\title{
MEDIDAS EM DIAGRAMAS TEMPO-FREQUÊNCIA APLICADO NA CARACTERIZAÇÃO DE TRANSIÇÕES DE ESTADO COM O USO DE SINAIS ULTRA-SÔNICOS RETROESPALHADOS DO PROCESSO DE COAGULAÇÃO SANGUÍNEA: UMA ANÁLISE NUMÉRICA
}

\author{
CARLOS DIAS MACIEL ${ }^{1}$ \\ LANA RIBEIRO DA FONSECA MONTEIRO*
}

MACIEL, CD.; MONTEIRO, L.R.F. Medidas em diagramas tempo-frequência aplicado na caracterização de transições de estado com o uso de sinais ultra-sônicos espalhados do processo de coagulação sanguínea: Uma análise numérica. Semina: Ci. Exatas/Tecnol. Londrina, v. 21 , n. 4, p. 35-45, dez. 2000.

RESUMO: Este trabalho apresenta uma forma alternativa de análise do sinal obtido a partir do Método Ultra-Sônico (US) no estudo das alterações do plasma sanguíneo em processo de coagulação. A ênfase da nova análise se baseia nos diagramas tempo $x$ frequência de sinais digitaiizados produzidos a partir de sinais de espalhamento US de partículas em movimentação aleatória. Para isso, foram produzidos 7 testes TP (tempo de protrombina) com plasma padronizado normal quanto aos fatores da coagulação e 1 deficiente de Fator $V$.

PALAVRAS-CHAVE: ultra-som, hemostasia, coagulação, análise tempo-frequência.

\section{INTRODUÇÃO}

O complexo processo de coagulação do sangue é de vital importância para preservar as funções do corpo humano. Este processo é iniciado por uma ativação externa e consiste em uma seqiuência de reações químicas. A falta da concentração adequada de um ou mais fatores necessários nesta cascata de reações resulta em uma inadequada formação do produto final, a fibrina.

Normalmente os testes clínicos que avaliam os mecanismos internos da cascata de coagulação são baseados na medida do tempo entre o inicio induzido da coagulação até a formação de fibrinas estáveis. Desvios do tempo normal de coagulação indicam que algum fator não esta adequadamente desempenhando as suas funções no processo e mais testes devem ser realizados para que este seja indicado.

Em Machado et al. (1997) foi apresentada uma análise de desempenho da estimativa do tempo total de coagulação medido com o uso de ultrasom. Neste trabalho foi feita uma comparação com dois outros métodos e os resultados obtidos foram satisfatórios. Os testes de coagulação realizados para balizar o processo ultra-sônico foram os teste de TP (Tempo de Protrombina) e TTAP (Tempo de Trombina Parcialmente Ativado), Monteiro (2000), sendo comparados pelos métodos manual e o fibrômetro.

$\mathrm{Na}$ Figura 1 é apresentado o diagrama esquemático da montagem experimental para o processo de medida do tempo de coagulação com o uso de ultra-som.

O princípio físico do funcionamento deste processo se baseia na medida do sinal retroespalhado por microesferas de vidro imersas no plasma sanguíneo contido na cubeta, Figura 1. Quanto menos viscoso for o plasma mais as microesferas de vidro (diâmetros de 0,074 a 0,105 mm) serão agitadas pelos 4 transdutores de ultra-som (4TT). Os transdutores que farão as medidas de agitação possuem frequência de $10,4 \mathrm{MHz}$ e possuem inclinação de 90 graus, de forma que o transdutor $\mathrm{Rx}$ recebe apenas os sinais retroespalhados pelas microesferas oriundos dos transdutor Tx. O sinal recebido em $R x$ é demodulado pelo mesmo sinal em Tx de forma que se obtém um sinal de

${ }^{1}$ Professor Adjunto do Departamento de Engenharia Elétrica/UEL. Caixa Postal 6044. CEP 86051-990 Londrina - PR, Fone (43) 371-4779 e-mail: <maciel@uel.br>.

${ }^{2}$ Mestre em Engenharia Biomédica pela COPPE/UFRJ. R. Luiz Gama, 494 - Ap. 101 - Parque Tietê, Sâo Joâo de Meriti - RJ - CEP 21.580-830, Fone: (21) $671-6183$ 
envóltoria com frequência muito mais baixa que a excitação dos transdutores Tx e Rx. Posteriormente este sinal é digitalizado em um osciloscópio com frequência de amostragem de $2,5 \mathrm{kS} / \mathrm{s}$.

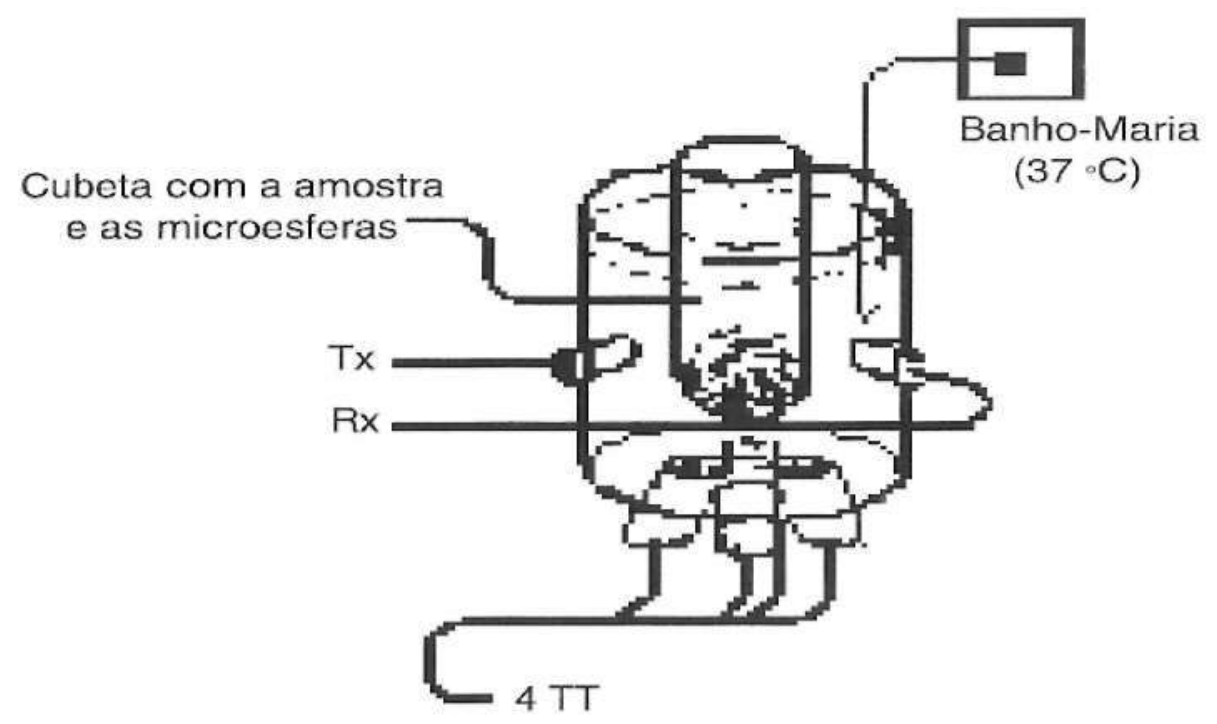

Figura 1 - Diagrama esquemático da montagem utilizada pelo Método Ultra-Sônico com a cubeta e as microesferas imersas.

Em Machado et al. (1999) é apresentada a modelagem do sinal retroespalhado pelas microesferas de vidro imersas em um meio viscoso a qual deriva uma expressão que relaciona a velocidade média quadrática das microesferas com a resposta tempo-frequência do sinal retroespalhado. A expressão final obtida é a eq.1.

$$
<n v_{y}{ }^{2}(\mathrm{t})>\infty \int_{-\infty}^{\infty} \omega^{2} \mathrm{~W}(\mathrm{t}, \omega) \mathrm{d} \omega
$$

onde: $\left\langle\mathrm{nv}_{\mathrm{y}}{ }^{2}>\right.$ é a velocidade média quadrática normalizada no plano formado pelos transdutores Tx e $R x$, e $W(t, \omega)$ é a resposta tempo-freqüência do sinal retroespalhado pelas microesferas. são:

As suposições para a derivação desta equação

1. todas as partículas são consideradas esféricas com mesmo raio e densidade;

2. A média das velocidades das partículas na direção y é zero pois, caso contrário, existiria o acúmulo de partículas em uma parte da cubeta;

3. As componentes horizontais de velocidade no plano y são independentes.

A suposição de que as microesferas de vidro apresentam o mesmo diâmetro e densidade não pode ser considerada válida pois existem impurezas entre as microesferas. A seleção da faixa de diâmetro usado no experimento é feito por peneiração.

Testes no laboratório usando novas cubetas de vidro borosilicato transparentes mostraram que a afirmativa 2 também não é valida pois existe um acumulo de partículas em uma determinada região das cubetas.

Embora muitos resultados experimentais tenham sido obtidos e estudos comparativos indiquem que este processo seja equiparado aos exames laboratoriais clássicos para a medida do tempo de coagulação, a modelagem desenvolvida ainda necessita ser refinada (Monteiro, 1998).

A proposta inicial do uso de ultra-som para caracterizar o plasma foi com o objetivo de estimar o tempo de formação da rede de fibrina. Posteriormente tentou-se inferir na qualidade do processo de formação das tramas de fibrina e possíveis deficiências químicas durante a coagulação. Para isso a análise do processo de coagulação considerou algoritmos de análise do sinal retroespalhado cada vez mais sofisticados. Trabalhos anteriores (Cordeiro (1998), Cardona (1995) e Machado et al. (1991)) realizam a medida direta da velocidade média quadrática normalizada, considerando uma simplificação do cálculo da distribuição tempo-frequência. O trabalho de Monteiro (2000) apresentou uma análise da determinação do tempo de coagulação pela interpretação direta dos diagramas tempofrequência. Outro aspecto positivo deste trabalho foram as interpretações de alguns resultados espúrios do processo.

Analisando-se os diagramas tempo-frequência observa-se que ao longo de toda escala temporal o espectro de potência possui a forma de $1 / f^{\mathrm{a}}$ (onde $f$ 
representa a frequência e a $>0$ ). Essa particularidade esta apresentada em diversos fenômenos físicos e ainda hoje é alvo de muitos estudos (Kaulakys (1999), Osborne \& Pastorello (1993), Theiler (1991) e Osborne \& Provenzalle (1989)).

Este trabalho tem a finalidade de apresentar uma forma alternativa de caracterização de sinais de coagulação sanguínea/plasmática coletados pelo Método US proposto em Machado et at. (1991) e Machado et al. (1997). Essa variante de análise aplica diagramas tempo x frequência na análise de sinais digitalizados, produzidos a partir de sinais de espalhamento US de microesferas de vidro em movimentação, imersas em amostras de plasma padrão normal.

No item Teoria será feita uma breve revisão dos métodos adotados para a estimativa do expoente a nos diagramas tempo-frequência. Também neste parágrafo serão feitas algumas suposições sobre os mecanismos de coagulação presentes durante o processo.

$\mathrm{Na}$ Metodologia será descrito o aparato experimental usado e também os algoritmos para o processamento de sinais adotados ao longo deste trabalho. No item Resultados são apresentados os gráficos comparando para a determinação do expoente a e gráficos, consolidando o resultado da análise de sete plasmas normais e um plasma deficiente em Fator V. Por ultimo, é feita uma discussão e conclusão dos resultados alcançados.

\section{TEORIA}

Series temporais com espectro de potência da forma $1 / f^{a}$ tem sido observadas em muitos resultados obtidos a partir de experimentos físicos, biológicos, econômicos, tecnológicos e sistemas sociológicos. Exemplos deste tipo de serie incluem dados geofísicos como medidas de índice pluviométricos e temperaturas, números de manchas solares, frequência de terremotos, variabilidade dos batimentos cardíacos, estrutura do DNA, texturas, imagens médicas e muitos outros.

O fato de que sinais da forma $1 / f^{a}$ sejam encontrados em diversos sistemas da natureza, nos conduz a especulação de que poderia existir algum mecanisrno genérico associado a esses processos. Uma abordagem para a caracterização desse mecanisrno genérico é tentar determinar para uma dada serie numérica o valor do expoente ex. Considerando que um processo genérico hipotético esteja ocorrendo durante a coagulação para a geração do sinal $1 / \mathrm{f}^{\mathrm{a}}$, espera-se que a monitoração de a indique alterações deste mecanismo e que a se altere ao longo do processo de coagulação.

Uma forma natural de fazer a estimativa de a e diretamente pelo espectro de potência. Essa abordagem consiste em se obter o quadrado do modulo da FFT (fast fourier transform) dos dados e interpolar uma reta com os pontos dados por $\ln \left\{S^{2}(f)\right\}$ por $\ln \{f\}$, onde $\ln \{$.$\} representa a função$ logaritmo. Considerando o espectro da forma $S^{2}(f)=C / f^{\infty}$ produzirá uma reta com equação

$$
\ln \left\{\mathrm{S}^{2}(\mathrm{f})\right\}=\text { Constante }-\alpha \ln \{\mathrm{f}\}, \quad \text { eq. } 2
$$

- expoente a será a inclinação da reta estimada por técnicas clássicas de regressão linear.

Posteriormente MaCoy et al. (1997) propuseram o uso do método de estimativa espectral multitaper (Thonsom, 1982) combinado com regressão linear para a estimativa de a. Para a estimativa espectral multitaper é adotado um conjunto de sequências ortogonais para a multiplicação com a série temporal inicial. Tem com principal vantagem a estimativa espectral resultante com menor variância.

Ambos os métodos citados possuem desvantagens quando usados diretamente:

1 . o espectro de potencia $\left(s^{2}(f)\right)$ possui frequências espaçadas em $f$ mas não em $\ln \{f\}$, levando a muitas estimativas em altas frequência e menos em baixas frequência. Desta forma, desloca-se a linha interpolada de forma a considerar mais os padrões gerados pelas altas frequências.

2. A transforrnação logarítmica não preserva a estrutura da variância das estimativas em cada frequência. Estimativas de frequência com grandes amplitudes possuem variância menor que a variância de frequências com menor amplitude.

Outras técnicas podem ser utilizadas para a estimativa do valor de a embora não seja objeto deste trabalho. Pode-se citar Peng et al. (1995) com o uso da DFA (detrended fluctuation analysis)

\section{METODOLOGIA}

A montagem experimental.

Os testes TP foram feitos empregando 7 alíquotas de uma mesma amostra de plasma padrão normal quanto aos fatores da coagulação. Tanto o plasma padrão quanto o plasma deficiente de Fator $V$ foram adquiridos da Sigma Aldrich ${ }^{3}$. Esses plasmas quando adquiridos estão na forma liofilisada e antes do uso são reconstituídos para serem aplicados nos experimentos.

${ }^{3}$ P.O.Box 14508, St. Louis, M063178 USA 
Os transdutores transmissores de potência (TT) utilizados para agitar as partículas ressonam em 2,72 $\mathrm{MHz}$ e foram excitados nesta frequência com potência $I_{\text {spta }}$ de $8,43 \mathrm{~W} / \mathrm{cm}^{2}$, e os transdutores de monitoração do processo (par Tx/Rx), em 10,4 $\mathrm{MHz}$. O sinal proveniente do transdutor $\mathrm{Rx}$ é demodulado de forma a se obter apenas a sua envoltória. A frequência de amostragem $\left(f_{a}\right)$ dos sinais de envoltória é de $2,5 \mathrm{kS} / \mathrm{s}$.

O processamento de sinais.

Os diagramas tempo-frequência foram obtidos a partir da análise de espectrograma usando janelas com 512 amostras. Quando se divide uma série temporal longa em segmentos menores implicitamente está se ajustando a menor frequência que se pode considerar em cada segmento. Caso se deseje considerar a frequência inferior (fi) nas análises a duração total de cada trecho deverá ser de $1 / \mathrm{fi}$ segundos ou $\mathrm{fa} / \mathrm{fi}$ amostras. A maior frequência segue a determinação do Teorema de Nyquist (fs fa/2).

Em cada janela dos diagramas tempofrequência a estimativa espectral foi implementada com o uso de duas 2 técnicas diferentes. A primeira implementação foi com o uso da Transformada Rápida de Fourier (FFT) e a segunda foi com a estimativa espectral usando multitaper. Em ambas as técnicas, o sinal total foi dividido em 512 amostras (fi $4,5 \mathrm{~Hz}$ ) com frequência superior de $1250 \mathrm{~Hz}$, aproximadamente 9 oitavas de sinal.

O tempo TP para a coagulação normal está na faixa de 11-13 segundos. A taxa de amostragem de $2,5 \mathrm{KS} / \mathrm{s}$ equivale a 27.500 amostras. O sistemas de coleta (osciloscópio Tektronics TDS 420) permite armazenar 60.000 amostras (aproximadamente 24 segundos de experimento). $\mathrm{Na}$ Figura 2 é apresentado um sinal típico de espalhamento de uttra-som durante o processo de coagulação do plasma normal.

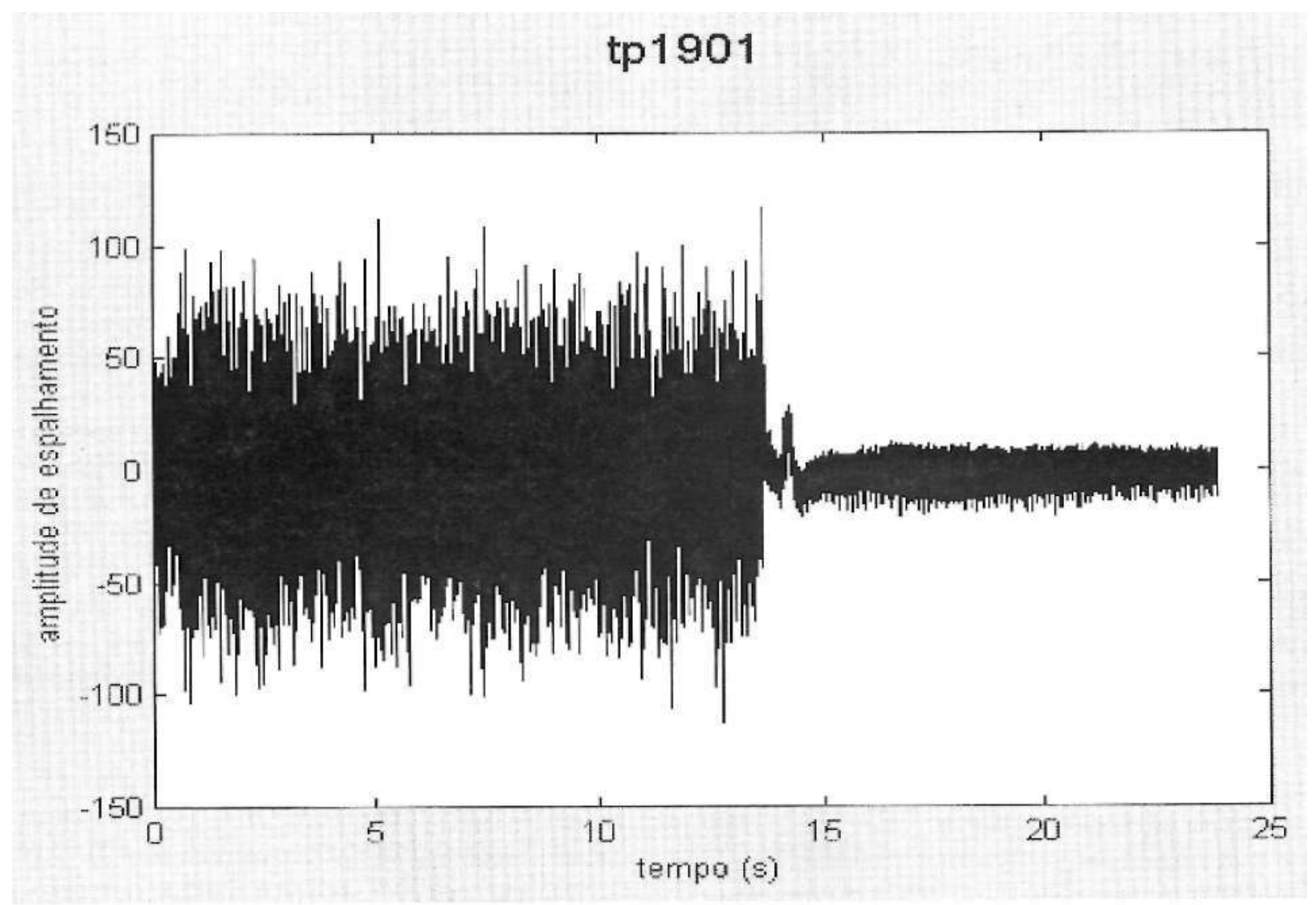

Figura 2 - Sinal retroespalhado típico por plasma sangüíneo normal.

Antes de se calcular o espectro de potência usando a FFT dos trechos do sinal, cada segmento é multiplicado por uma janela para melhorar a sua estimativa. Ao longo deste trabalho foi adotado a janela de Hanning (Therrien, 1992).

\section{RESULTADOS}

$\mathrm{Na}$ Figura 3 superior esta apresentado o diagrama do espectro de potência ao longo do tempo. Observa-se que no início do processo de 
coagulação o sinal espalhado possui um conteúdo harmônico muito diferente do sinal após a coagulação (próximo a 13 segundos).

Podem ser observadas linhas horizontais no diagrama tempo x espectro de potência. Essas linhas são interferências causadas pela rede elétrica e combinações das frequências usadas para excitar os transdutores de agitação e de monitoração.

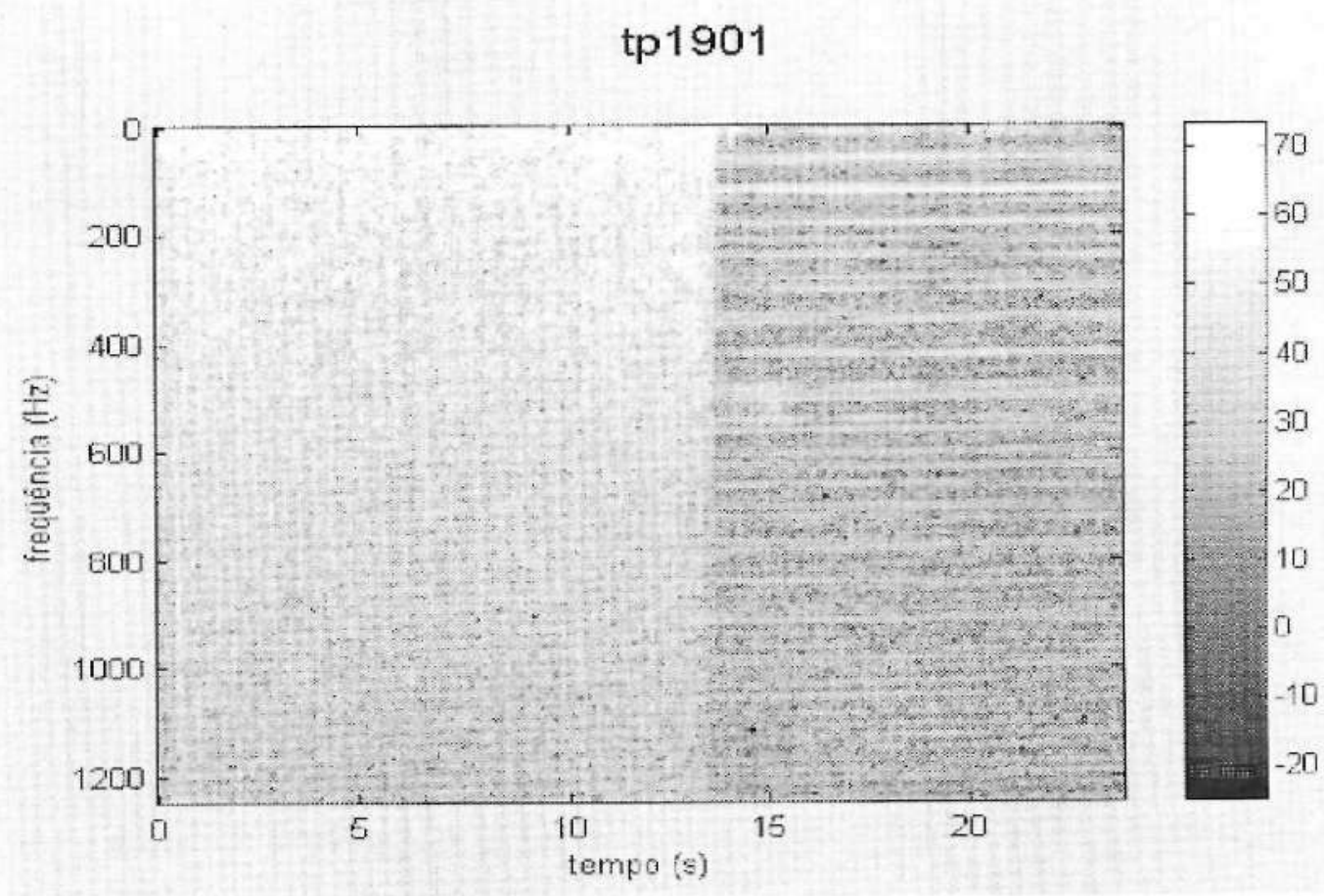

Figura 3 - Diagrama Tempo-freqüência usando FFT do sinal apresentado na Figura 2.

Na Figura 4 são apresentados dois exemplos de retas interpoladas no logaritmo de espectro de potência em duas posições temporais diferentes.
A janela temporal da Figura superior esta em 1,94 segundos e a inferior em 16,28 segundos.

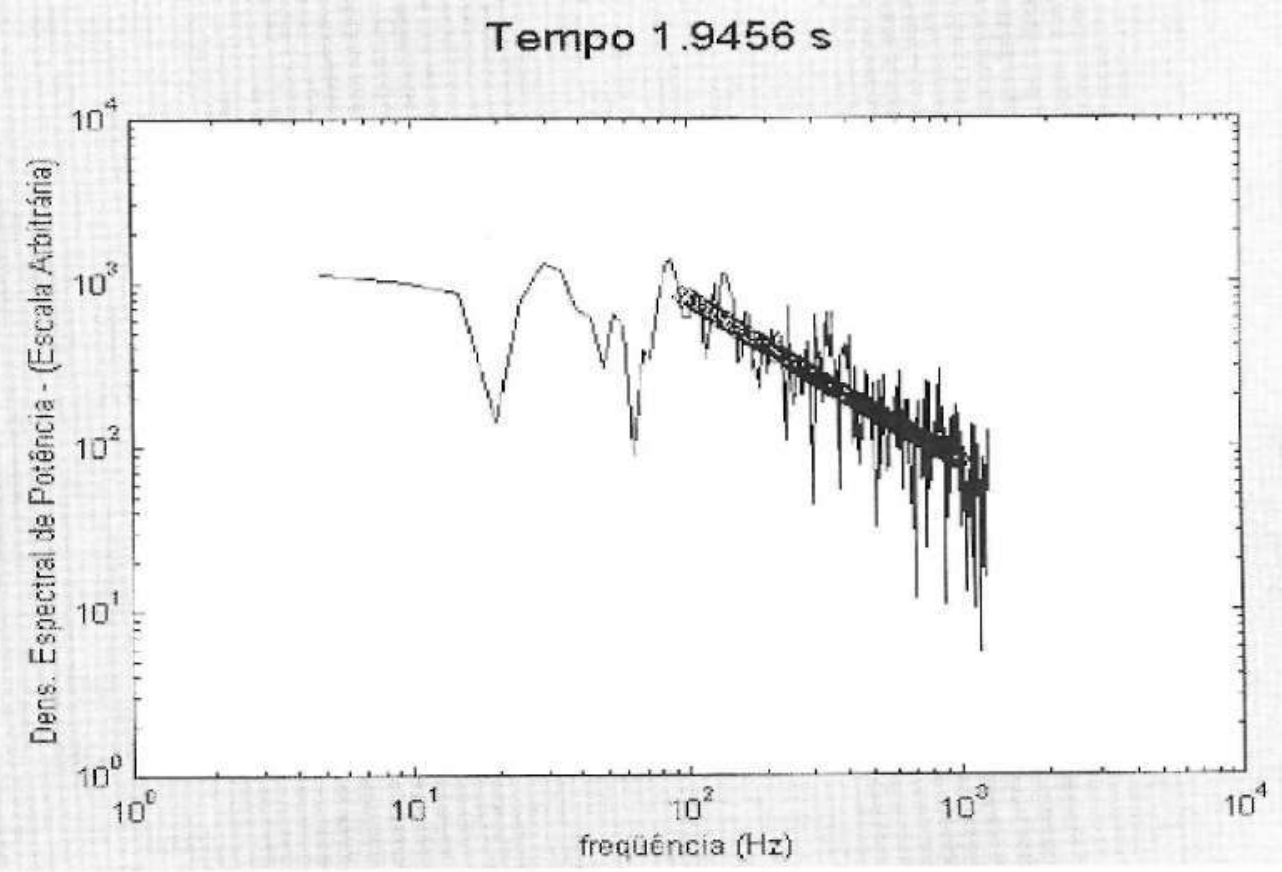

Figura 4 -Retas interpoladas no espectro de potência calculado com FFT em duas posições temporais diferentes. Superior em 1,94 segundos e a inferior em 16,28 segundos. 
Os coeficientes angulares para cada janela temporal ao longo de todo processo de coagulação sanguínea usando FFT esta mostrado na Figura 6. Na Figura 8 é o mesmo gráfico embora usando estimativa espectral multitaper. Observa-se dois estados distintos: o primeiro quando as microesferas estão no meios viscoso e posteriormente quando presas pela rede de fibrina. Observa-se também, antes da transição, uma alteração do coeficiente angular indicando que o meio ficou menos, viscoso. Sua interpretação será feita no item Discussão e Conclusão.

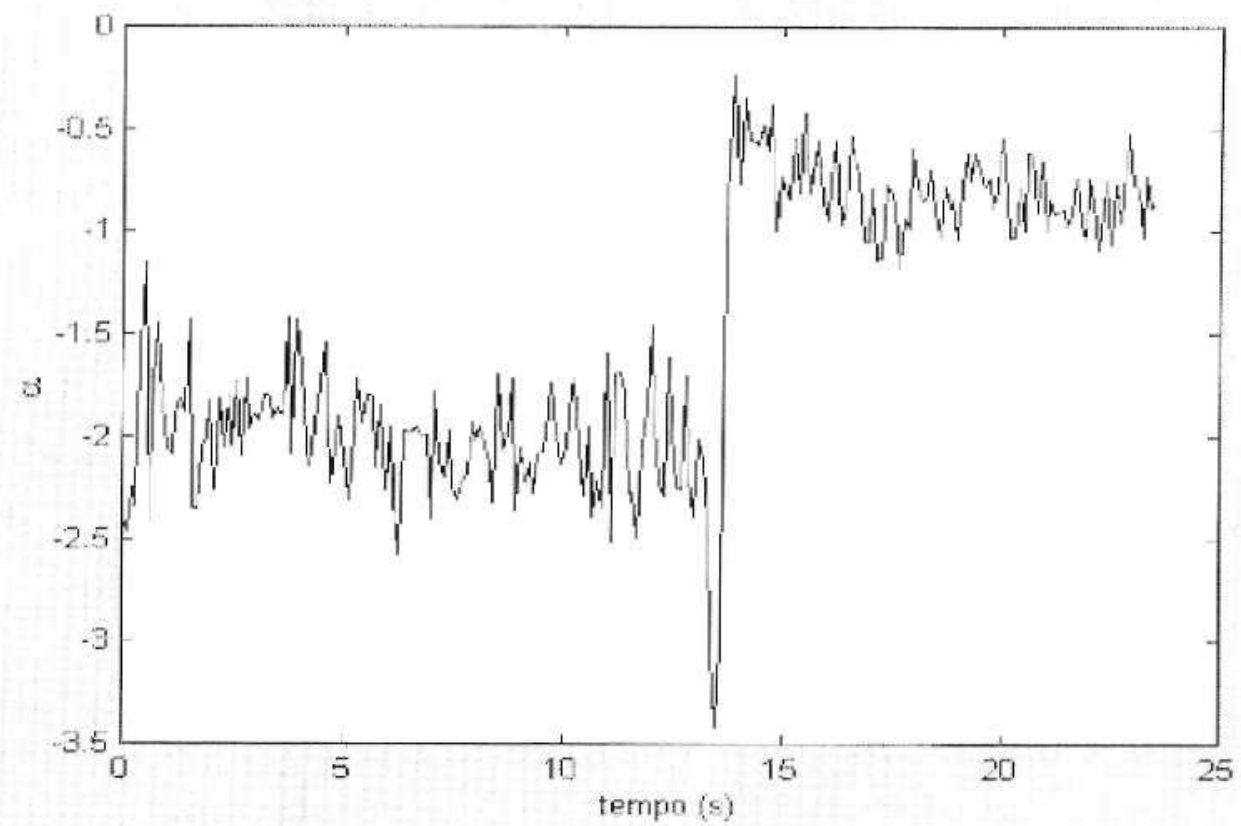

Figura 5 - Coeficientes angulares para cada janela temporal calculado ao longo de todo processo de coagulação sangüinea e usando estimativa espectral FFT.

Observam-se nas Figuras 6 e $\quad 8$ uma representação alternativa (Diagrama de Pontos) para os coeficientes angulares apresentados nas Figuras 5 e 7 . Cada par de pontos consecutivos (i, i+1) nas Figuras 5 e 7 são representados como um ponto dos diagramas mostrados nas Figuras 6 e 8. Observam-se dois cluster distintos, associados com os estados do plasma. Quanto mais agrupados se apresentarem os pontos, menos variação do coeficiente angular estará presente.

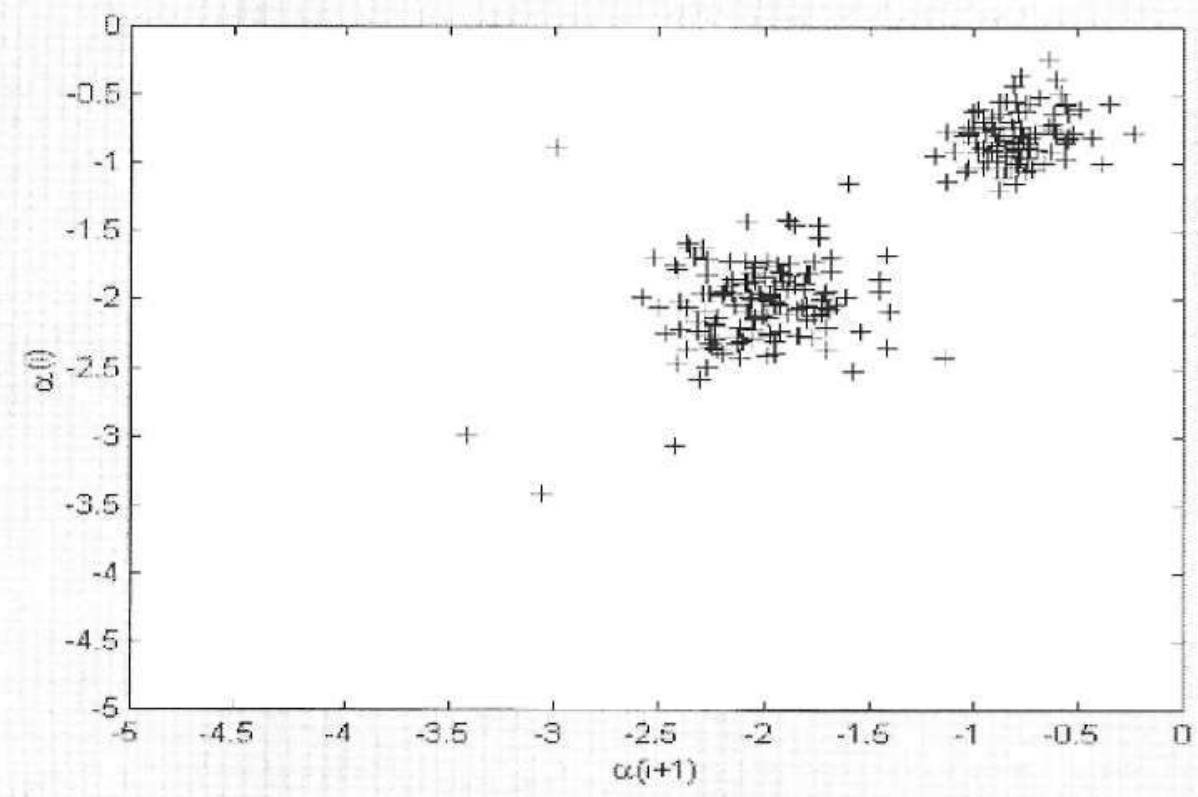

Figura 6 - Diagrama de Pontos usando a estimativa espectral FFT. 


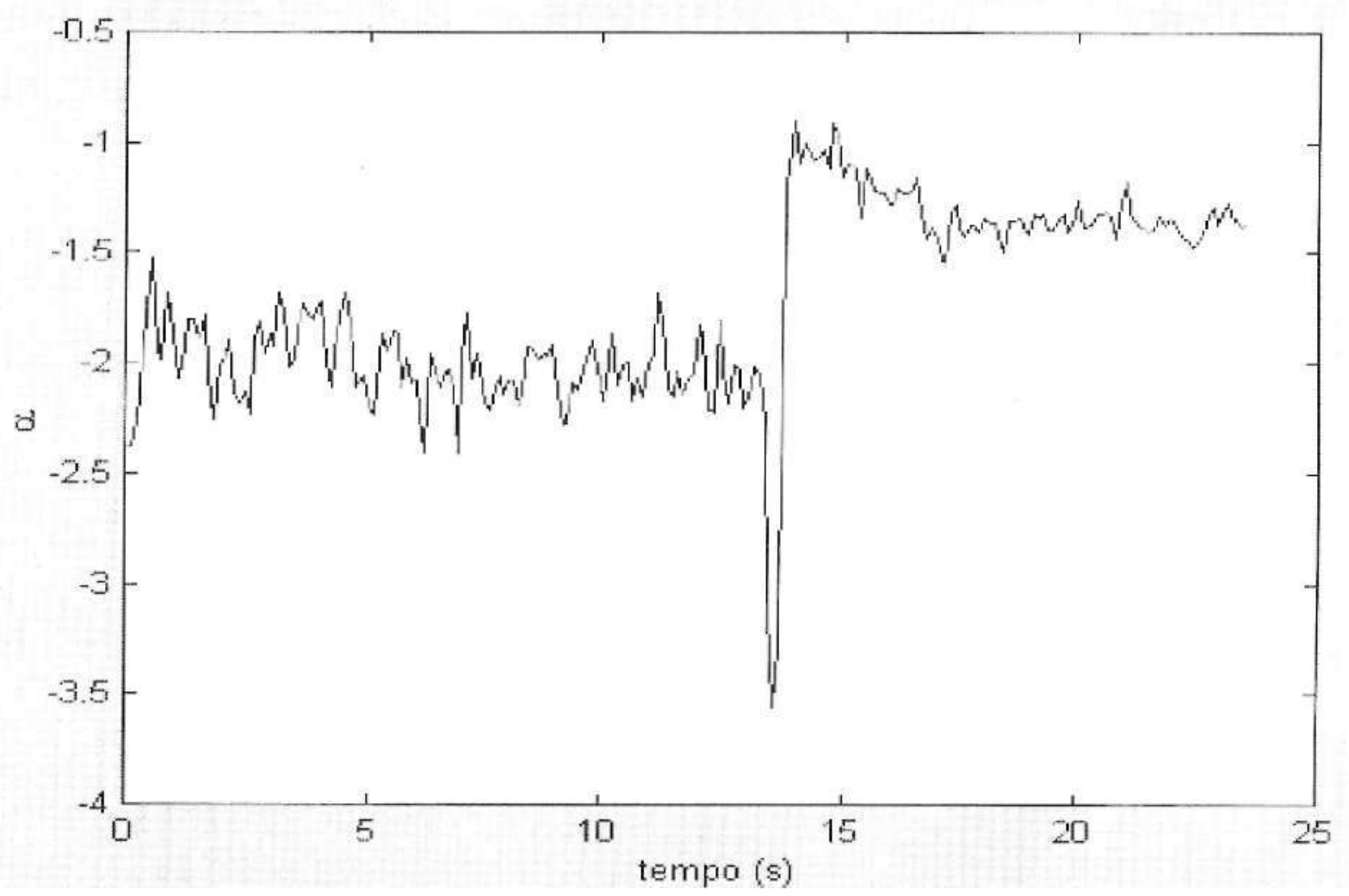

Figura 7 - Coeficientes angulares para cada janela temporal calculados ao longo de todo processo de coagulação sangüínea e usando multitaper.

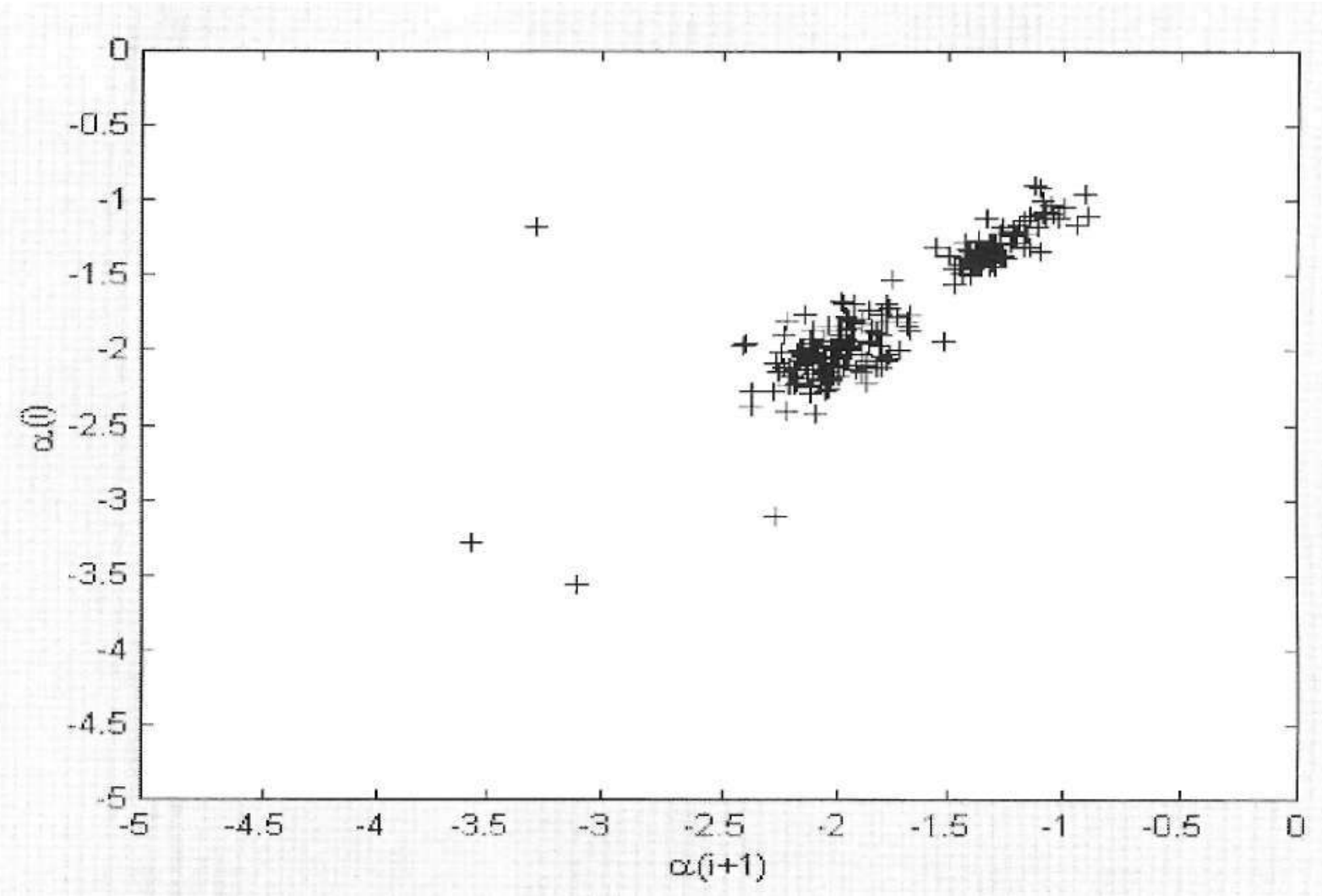

Figura 8 - Diagrama de Pontos usando a estimativa espectral multitaper (Figura 7). 
Na Figura 9 é apresentado o resultado da estimativa do coeficiente angular a para os 7 casos de teste normais. Observa-se de forma qualitativa que existem dois cluster, indicando os dois estados que se encontram o plasma sanguíneo. Na é apresentado um gráfico de histograma com 40 classes modais. Observa-se que durante o estado livre as microesferas geram uma dispersão maior de a e que durante a fase de coagulo essa dispersao é menor.

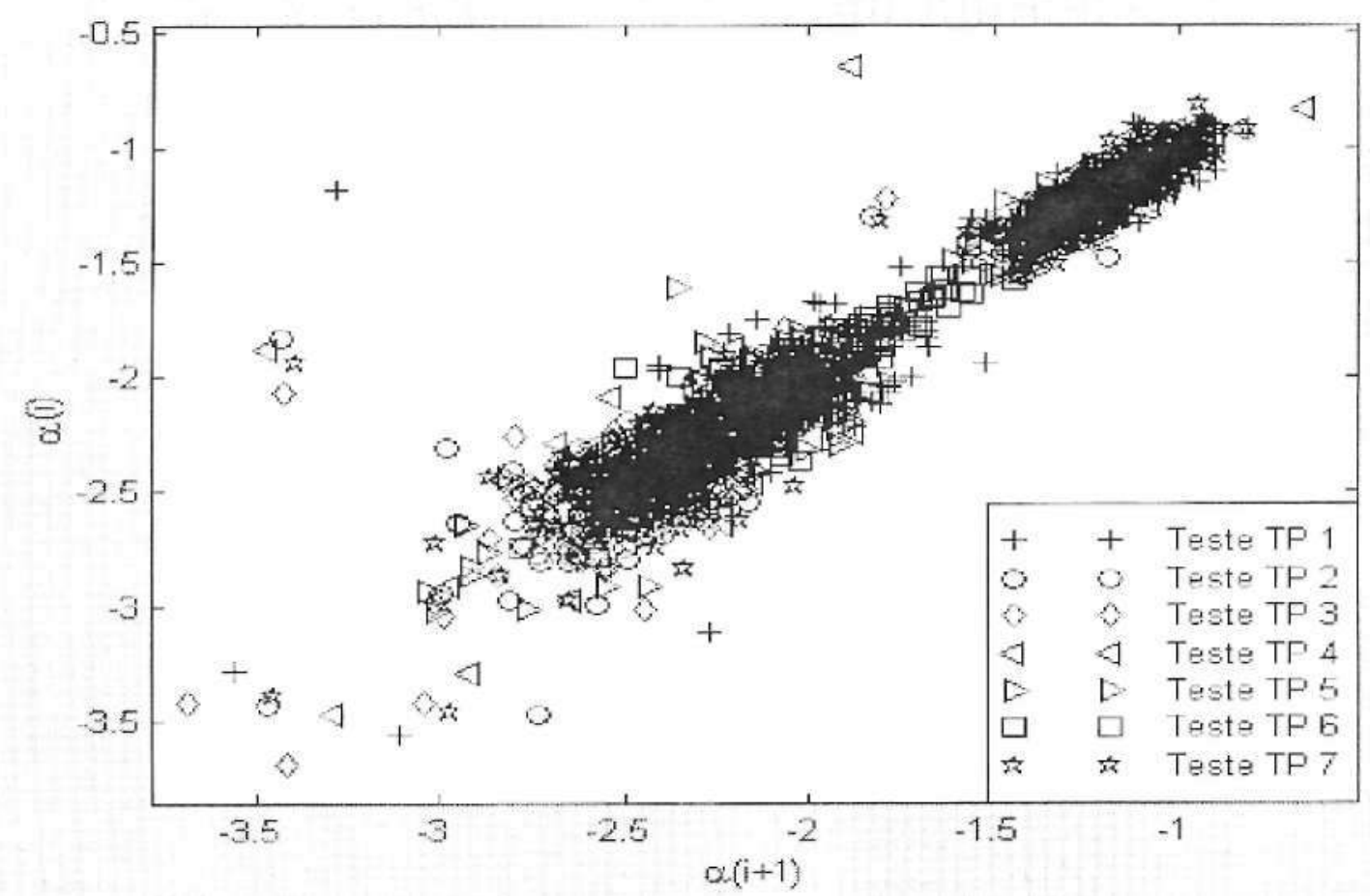

Figura 9 - Resultado da estimativa do coeficiente angular $\alpha$ para os 7 casos de teste normais usados para avaliar o processo. Os valores de $\alpha$ foram calculados pela estimativa espectral multitaper.

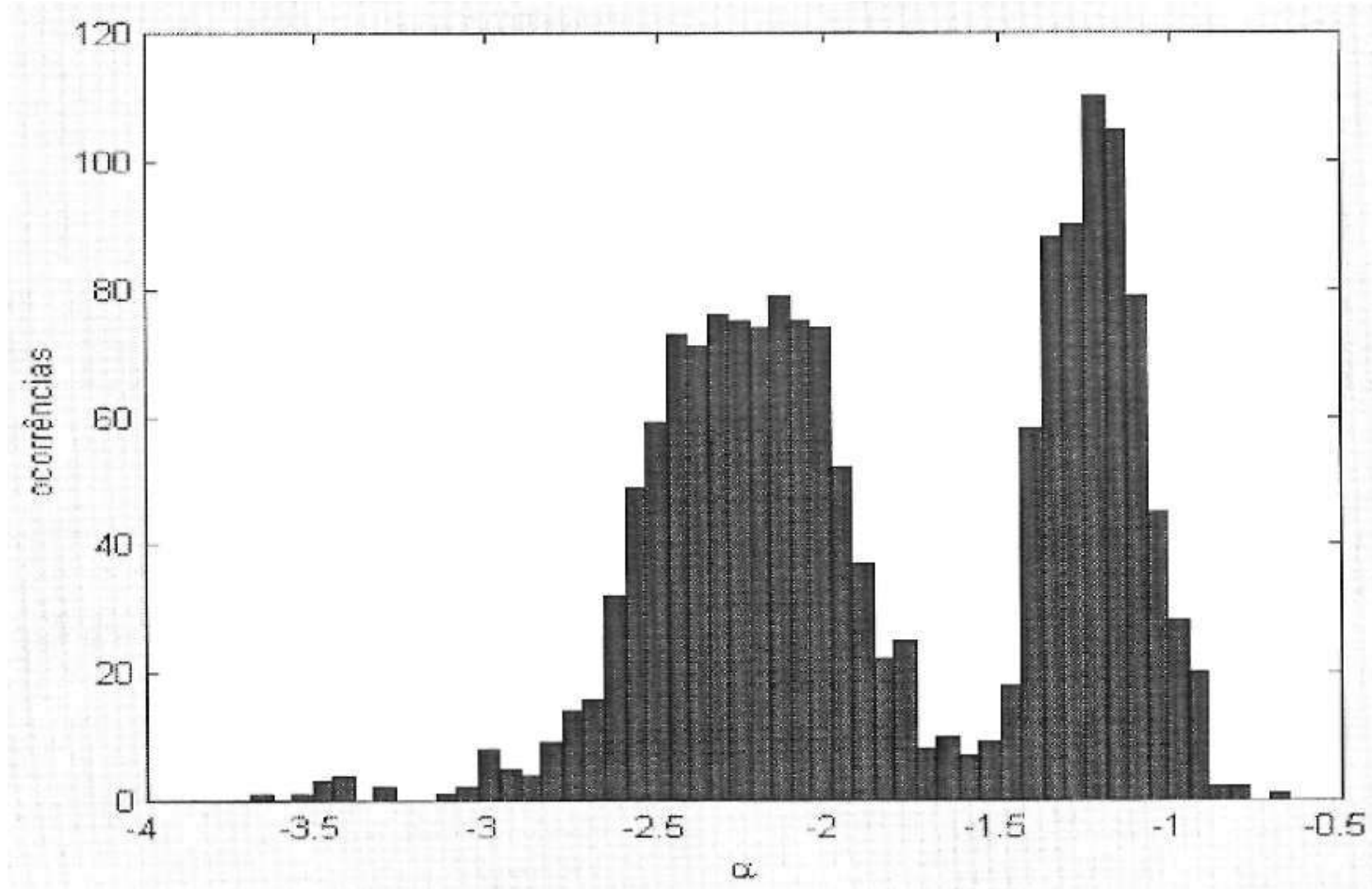

Figura 10 - Histograma com os valores de $\alpha$ separados om 40 classes modais. Observam-se os dois estados que se encontram os plasmas ao longo da coagulaçăo. 
A Figura 11 representa o sinal retroespalhado pelas microesferas imersas em um plasma deficiente em Fator $V$ cuja representação tempofrequência é mostrado na Figura 12. Esse plasma é do tipo hemofílico e o processo de coagulação é mais lento. O gráfico da Figura 11 esta com a escala de tempo deslocada e, desta forma, são apresentados apenas os últimos 24 segundos de coleta do sinal.
Na Figura 12 se apresenta o diagrama tempofrequência, observa-se que próximo da coagulação as frequências mais altas são atenuadas mais rapidamente que as demais. Na Figura 13 está o valor de a ao longo do processo de coagulação. Observa-se que momentos antes da coagulação ocorre uma depressão com duração muito maior que nos casos de plasma normal (Figura 7).

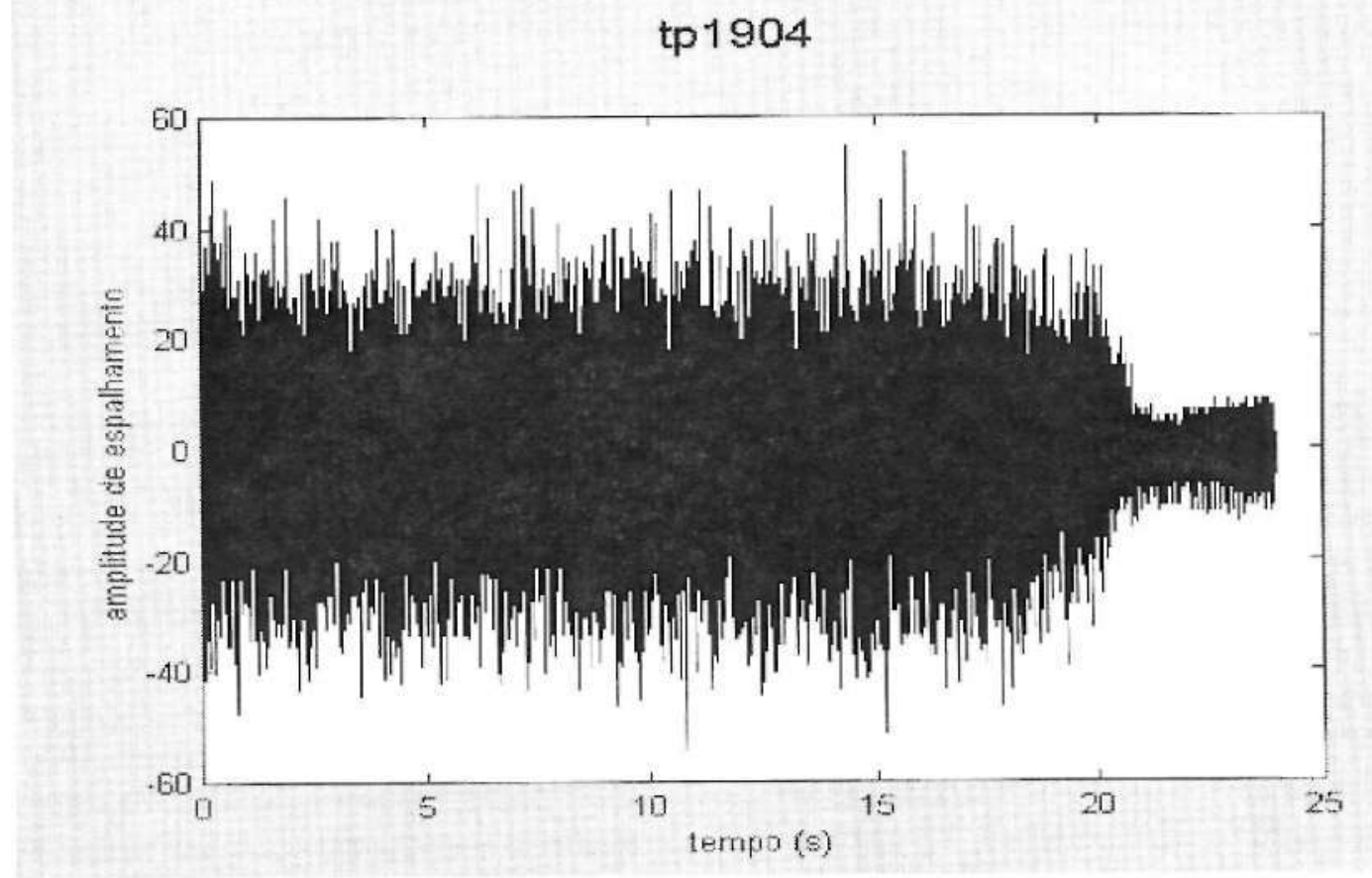

Figura 11 - Sinal retroespalhado por plasma sangüíneo deficiente de Fator V.



Figura 12 - Diagrama Tempo-freqüência usando FFT para o plasma deficiente de Fator V. 


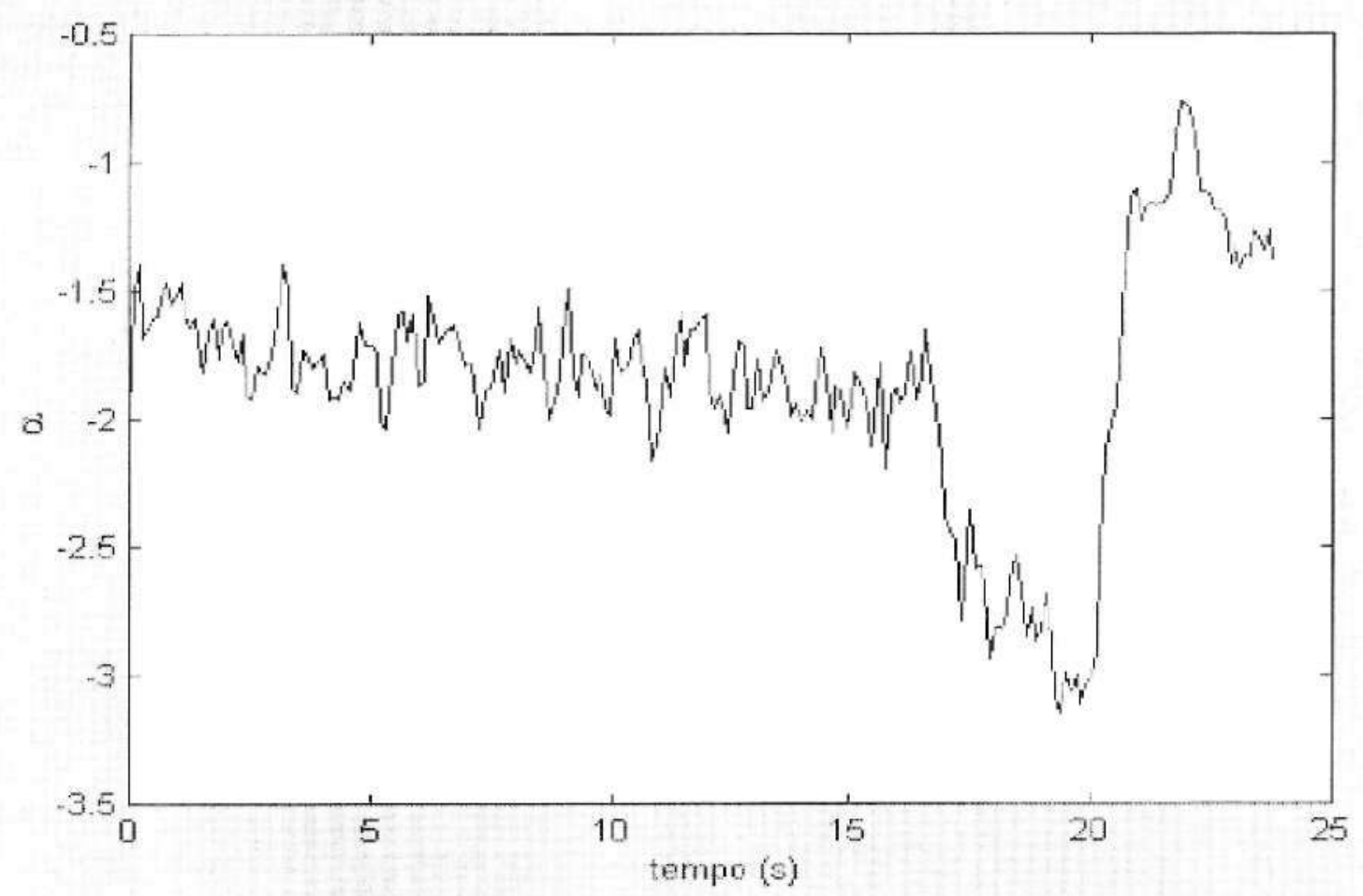

Figura 13 - Coeficientes angulares para cada janela temporal calculados ao longo de todo processo de coagulação sangüínea deficiente de Fator $\mathrm{V}$ usando a estimativa multitaper.

\section{DISCUSSÃO E CONCLUSÕES}

Os testes feitos durante o desenvolvimento desta análise apresentou que a estimativa espectral multitaper levou a resultados com menor variância de a. A estimativa do valor de a para urn plasma padrão normal antes da coagulação foi em torno de $-2,3$ e após a coagulação era próximo de $-1,2$.

A maior ou a menor facilidade que as microesferas encontram para navegar no plasma irradiado pelo US está relacionada ao tipo de obstáculo que essas partículas enfrentam em sua trajetória. Durante o período em que o plasma, enquanto colóide, sofre as primeiras reações do processo de coagulação, as macromoléculas que compõem sua fase dispersa vão sendo recrutadas e saem da suspensão, tornando o meio menos viscoso. Nessafase, o valor de a experimenta uma queda no seu valor, visto que há uma facilidade maior de locomoção das microesferas, podendo registrar um pico na proximidade do instante em que há a coagulação. Todavia, a medida em que as primeiras tramas da rede de fibrina vão surgindo, o meio começa a oferecer uma certa resistência ao trânsitodas partículas, bloqueandoIhes completamente o caminho no instante em que há a polimerização dos monômeros de fibrina e o coágulo se consolida. Desta feita, o sinal sofre a queda em etapas registrada na Figura 2.
Os diagramas tempo $x$ frequência revelam ainda que as frequências mais altas caem primeiro, o que poderia estar indicando que as microesferas estariam sendo agrupadas em classes, de acordo com sua capacidade de manter sua movimentação diante do meio que esta se tornando mais viscoso. Talvez não se possa dizer que essa capacidade esteja relacionada ao tamanho da partícula, uma vez que são essas mesmas frequências que caem primeiro e as últimas que desaparecem. Por outro lado, talvez sejam as partículas de menor diâmetro que, por serem menores, sofram com mais intensidade os possíveis choques com as tramas de fibrina que estão se formando e se polimerizando, enquanto as de maior diâmetro, ou nâo sentem tanto esses choques, ou são capazes de romper tais tramas, enquanto ainda instáveis, liberando o caminho daquelas de menor diametro e permitindo-Ihes que se movimentem por mais tempo.

Tendo em vista que os resultados obtidos coadunam com os esperados e Ihes acrescentam informações, conclui-se que a aplicação deste recurso na análise dos sinais de coagulação sanguínea / plasmática humana possui grande valor, uma vez que possibilita um estudo mais profundo dos eventos que tomam parte nesse processo, contribuindo para uma maior compreensão dos mesrnos. 
MACIEL, CD., MONTEIRO, L.R.F. Measurements in time-frequency diagrams employed in the characterization of state transitions using scattered ultrasound signals during blood clotting: a numerical analysis. Semina: Ci. Exatas/Tecnol. Londrina, v. 21, n. 4, p. 35-45, dez. 2000.

ABSTRACT: This work introduces a new perspective of analyzing the signal obtained by means of the Ultrasonic Method (US) in the study of blood plasma alterations during coagulation. The emphasis is based on time versus the frequency of digitalized signals generated by US particles scattering in random motion. For this purpose, seven PT (protombin time) tests were produced with normal coagulation plasma samples and a deficient one in Factor $V$.

KEY WORDS: ultrasound; hemostasis; coagulation; time-frequency analysis.

\section{REFERENCIAS BIBLIOGRAFICAS}

CARDONA, M. A. R. Método ultrasônico para monitoração do alteraçöes da reologia do plasma sangüineo durante a coagulaçāo. 1995. Tese (M.Sc.) - UFRJ/COPPE/PEB, Rio de Janeiro, 1995.

CORDEIRO, A. P. Q. Caracterização reológica do tempo de tromboplastina parcial ativado, utilizando o método ultra-sônico. 1998. Tese (M.Sc.) - UFRJ/COPPE/PEB, Rio de Janeiro, 1998.

KAULAKYS, B. Autoregressive model of $1 /$ f noise. Physics Letter A, v. 257, n. 1/2, p. 37-42, 1999.

MACHADO, J. C.; LENZI, A.; ALVES, C.H.F.; SIGELMANN, R. A. Analysis on the motion of particles by ultrasound and immersed in a host medium, with application to viscosity measurement. Acustica-Acta Acustica, v. 85, p. 816-824, 1999.

MACHADO, J. C.; LENZI, A.; SILVA, W. G.; SIGELMANN, R. A. An ultrasonic method to measure human plasma coagulation time. J. Acoust. Soc. Am., v. 90, n. 4, p. 1749-1753, 1991.

MACHADO, J. C.; VON KRUGER, M. A.; ALMEIDA FONTES, E. M.; De Almeida, M. M. G. Evaluation of an ultrasonic method applied to the measure of blood coagulation time. Physiol. Meas., v. 18, p. 129-143, 1997.

McCOY, E. J.; WALDEN, A.T.; PERCIVAL, D.B. Mutitaper spectral estimation of powor law process. [S.I.]: Imperial Colige of Science/Dept. of Mathematics, 1997. Technical Report TR 95-17.
MONTEIRO, L. R. F. Análise do sinal ultra-sónico de monitoração da coagulação do plasma sanguineo por diagramas tempo $x$ freqüência. 2000. Tese (M.Sc.) - UFRJt COPPE/PEB, Rio de Janeiro, 2000.

MONTEIRO, L. R. F.; Pereira, W. C. A. Método ultra-sônico de monitoraçāo da coagulação do plasma sanguíneo: uma análise crítica. In: FÓRUM NACIONAL DE CIÊNCIA E TECNOLOGIA EM SAÚDE, 4., 1998, Curitiba. Anais... Curitiba, out. 1998. p. 377-378.

OSBORNE, A. R.; PASTORELLO, A. Simultaneous ocurrence of low dimensional chaos and colored random noise in nonlinear physical system. Physics Letter A, v. 18, p. 153-171, 1993.

OSBORNE, A.R.; PROVENZALLE, A. Finite correlation dimension for stochastic systems with power law spectra. Physica D, v. 35, p. 357-381, 1989.

PENG, C. K.; HAVLIN, S.; STANLEY, H. E.; GOLDENBERGER, A. L. Detrend fluctuation analysis. Chaos, v. 5, n. 1, p. 82-87, 1995.

THEILER, James. Some comments on the correlation dimension of $1 / f \alpha$, noise. Physics Letter A, v.155, n. 8/9 (1991).

THERRIEN C. W. Discrete random signals and statistical signal processing. [S.I.]: Prentice Hall, 1992. Signal Processing Series.

THOMSON, D. J. Spectrum estimationand harmonic analysis. Proc. IEEE, v. 70, p 1055-1096, 1982. 